that a fairly large number of patients in the University Clinic of Vienna would have met inclusion criteria. It is possible that other patients-related factors may have been involved in accounting for the very low sample size. In this respect, a wide gap between mainstream psychiatrists' views and patients' views regarding the usefulness of ECT has been revealed in a systematic review. ${ }^{6}$

1 Gryglewski G, Baldinger-Melich P, Seiger R, Godbersen GM, Michenthaler P, Klöbl M, et al. Structural changes in amygdala nuclei, hippocampal subfields and cortical thickness following electroconvulsive therapy in treatmentresistant depression: Iongitudinal analysis. Br J Psychiatry 2019; 214: 159-67.

2 Lagace DC, Eisch AJ. Mood-stabilizing drugs: are their neuroprotective aspects clinically relevant? Psychiatr Clin N Am 2005; 28: 399-414.

3 Sackeim HA, Prudic J, Fuller R, Keilp J, Lavori PW, Olfson M. The cognitive effects of electroconvulsive therapy in community settings. Neuropsychopharmacology 2007; 32: 244-54.

4 Fava GA. Can long-term treatment with antidepressant drugs worsen the course of depression? J Clin Psychiatry 2003; 64: 123-33.

5 Fava GA, Offidani E. The mechanisms of tolerance in antidepressant action. Prog Neuropsychopharmacol Biol Psychiatry 2011; 35: 1593-602.

6 Rose D, Fleischmann P, Wykes T, Leese M, Bindman J. Patients' perspectives on electroconvulsive therapy: systematic review. BMJ 2003; 326: 1363.

Marco Chiesa, Consultant Psychiatrist and Visiting Professor, University College London, UK. Email: m.chiesa@ucl.ac.uk

doi:10.1192/bjp.2019.232

\section{Response letter to the article by Killackey et al (2019) 'Individual placement and support for vocational recovery in first-episode psychosis: randomised controlled trial'}

It was with interest we read the results of the recent paper by Killackey et al evaluating the efficacy of individual placement and support (IPS) in first-episode psychosis (FEP) measured towards vocational outcomes and were excited to see positive results at earlier outcome points. ${ }^{1}$ The authors kindly offer multiple explanations of a loss of significance of such interventions at later end points, which we would like to contrast and expand upon.

The authors concluded that the research demonstrated a case for IPS in helping those with FEP return to work, despite this not being maintained at later time points. However, this may be optimistic given the key demographics examined could be potentially an overestimation.

First, the method of selection and randomisation denotes generous selection criteria but fails to fully account for an unequal distribution of affective presentations between group strata and self-selection of willing participants, suggesting a lower baseline functional impairment.

The evidence provided suggests short-term efficacy of IPS in motivated individuals of native language, with less severe symptoms, within a 'schizonormative' group and within a well-funded system. This is in keeping with previous research, but provides a relatively narrow margin for intervention, which does not clearly explain how and why it would be effective in those failing to make vocational recovery during usual treatment.
We would consider that those failing in the usual treatment arm may lie outside of the actionable group demonstrated, attributable to the coexistence of affective subtypes, more severe disease (omitted from the study), reduced language efficacy or other factors. Although some of this has evened out at later end-points, this may be because of other factors (remission of anergia, efficacy of pharmacological intervention). We would please request the authors address the above assertion.

Although the study does demonstrate some efficacy with those with a mood-affective component, as well as comorbid anxiety and post-traumatic stress disorder, unless further information is made available, it does not extrapolate the relevance of these factors into short- or long-term outcomes in a comprehensive way. We note that the control group included more affective presentations, which we hope have been accounted for.

The authors also comment that as a result of previous work, those operating in clinics may have been previously upskilled in IPS, which may also explain the high vocational rates ascribed to the treatment as usual arm and could have nullified significance at later end points. We would proffer an additional explanation, where some observer biases inherent in those clinics sharing both intervention and test cases would inspire more supportive treatment.

Finally, the measure of a successful outcome used may overestimate the success of the project, and the attenuation of employment in the intervention arm may further explain the loss of significance at later end points. We posit this could potentially empower the government into seeking more zero-hour contracts in the UK as evidence of employment. However, far from being a negative, it does suggest that IPS, and work around it, does favour positive outcomes and this has been reflected in long-term follow-up in a similar study in Switzerland ${ }^{2}$ and shown to improve outcomes. This suggests moreover that a team approach to support, regardless of vocational expert inclusion, may be the really successful intervention.

It may be that these programmes would present a viable intervention if tailored to adjust for affective symptoms, be maintained beyond 6 months, be 1:1, ${ }^{3}$ account for heterogeneity in comorbid substance use, account for patient aspirations, allow for differences between Australia and UK service structures and funding prospects, and include a team-based approach to service delivery.

1 Killackey E, Allott K, Jackson HJ, Scutella R, Tseng YP, Borland J, et al. Individual placement and support for vocational recovery in first-episode psychosis: randomised controlled trial. Br J Psychiatry 2019; 214: 76-82.

2 Hoffmann H, Jäckel D, Glauser S, Mueser KT, Kupper Z. Long-term effectiveness of supported employment: 5-year follow-up of a randomized controlled trial. Am J Psychiatry 2014; 171: 1183-90.

3 Holt RIG, Gossage-Worrall R, Hind D, Bradburn MJ, McCrone P, Morris T. Structured lifestyle education for people with schizophrenia, schizoaffective disorder and first-episode psychosis (STEPWISE): randomised controlled trial. Br J Psychiatry 2019; 214: 63-73.

B. M. Janaway, Core Psychiatric Trainee, National Hospital of Neurology and

Neurosurgery, UCLH, UK; Mukesh Kripalani, Consultant Psychiatrist, ADHD centre and PPCS, UK. Email: benjamin.janaway@nhs.net

doi:10.1192/bjp.2020.31 